\title{
Gamificando el aula: aplicación de una escape room colaborativa online
}

\author{
Vanesa Paula Cuenca-Gotor ${ }^{1}$, Alicia Herrero-Debón ${ }^{1}$, Santiago Moll-López ${ }^{1}$, Juan \\ Antonio Monsoriu-Serra ${ }^{1}$, José Antonio Moraño-Fernández ${ }^{1}$, Luis M. Sánchez Ruiz ${ }^{1}$ \\ y Erika Vega-Fleitas ${ }^{1}$ \\ ${ }^{1}$ Universitat Politècnica de València
}

\begin{abstract}
The arrival of the COVID-19 pandemic has led to an important change in the methodologies used in education. In this work the application of a virtual escape room is presented in the subject of Orbits, Satellites and Relativity in order to increase motivation and the acquisition of knowledge. The inclusion of game elements in the classroom has had a positive impact on the opinion and performance of students. The platforms used have been PoliformaT and TEAMS.
\end{abstract}

Keywords: escape room, PoliformaT, Game Based Learning, UPV.

\begin{abstract}
Resumen
La llegada de la pandemia de COVID-19 ha supuesto un cambio importante en las metodologías empleadas en educación. En este trabajo se presenta la aplicación de una escape room virtual en la asignatura optativa de Órbitas, Satélites y Relatividad con el fin de aumentar la motivación y la adquisición de conocimiento. La inclusión de elementos de juego en el aula ha tenido un impacto positivo en la opinión y en el rendimiento de los estudiantes. Las plataformas empleadas han sido PoliformaT y TEAMS.
\end{abstract}

Keywords: escape room, PoliformaT, Game Based Learning, UPV.

\footnotetext{
* Proyecto financiado por el Proyecto de Innovación y Mejora Educativa (PIME), Convocatoria A+D 2019, Código 1699-B. Universitat Politècnica de València
} 


\section{Introducción}

La reciente pandemia mundial por COVID-19 ha supuesto un cambio radical en el ámbito educativo. La adaptación a una metodología de enseñanza online ha supuesto un reto importante para los centros educativos y para todos los elementos de su comunidad. Las herramientas digitales empleadas para suavizar el cambio y mantener el contacto entre docentes y estudiantes han permitido también un cambio metodológico más profundo en la forma de enseñar. El empleo de estas herramientas digitales ha mejorado muchas habilidades de nuestros estudiantes y docentes, y se ha extendido su uso para la planificación y administración del proceso educativo. Se ha podido observar que las herramientas digitales y los recursos que se han generado permiten al estudiante un control significativo del proceso de aprendizaje, complementando los conocimientos con muchas fuentes educativas. Sin embargo, uno de los elementos importantes en el proceso de aprendizaje de nuestros estudiantes es la motivación. Esta se ha visto además afectada por el cambio de metodología, y es importante mantener o incorporar elementos que puedan mejorarla o incrementarla. Con respecto a las áreas de ciencia, tecnología, ingeniería y matemáticas (STEM), existía un grado alto de desmotivación previo (Borrego y col. 2017,Ross y Bell 2019), que la educación online, al menos en sus primeras fases de adaptación, no ha podido mejorar.

La incorporación de elementos de gamificación, en el ámbito educativo y en el desarrollo de las clases, ha dado buenos resultados: aumenta las tasas de éxito, de rendimiento y la motivación de los estudiantes (Bybee 2013, Mellado y col. 2014). El objetivo de emplear estos elementos de gamificación, y un aprendizaje basado en juegos, es crear un ambiente emocional positivo en el que el proceso de enseñanza-aprendizaje sea más atractivo e inclusivo, aumentando la motivación de los estudiantes, no solo mientras se participa en este proceso sino extendiéndolo al entorno fuera del aula (F. Sanchez-Martín J. C.-C. y Davila-Acedo 2017, Zamora-Polo, Corrales-Serrano y col. 2019, Papastergiou 2019).

La gamificación se puede definir como el uso de elementos de juego y técnicas de diseño de juegos en contextos ajenos al juego, como en la enseñanza (Deterding y col. 2011), para fomentar la participación activa mientras se resuelven problemas (De-Marcos y col. 2014, Warmelink y col. 2020, Zamora-Polo, Corrales-Serrano y col. 2019 y J. Sanchez-Martín, Corrales-Serrano y col. 2020). La gamificación, a diferencia del Game Based Learning (GBL), no se limita al ámbito educativo (de hecho, su origen está relacionado con áreas económicas y financieras (Deterding y col. 2011; F. Sanchez-Martín J. C.-C. y Davila-Acedo 2017), mientras que GBL se centra en el diseño de juegos en el entorno educativo (Ebner y Holzinger 2007; Papastergiou 2019). Antes de la pandemia, las técnicas de GBL se estaban aplicando con éxito en el ámbito educativo (Menon y Romero 2020; L. Rodrigues, Oliveira y H. Rodrigues 2019) y pueden adaptarse perfectamente en las metodologías educativas post-COVID. Además del aumento de la motivación (Deterding 2012; Fotaris y Mastoras 2019; Hamari y col. 2016), un beneficio muy interesante obtenido mediante la aplicación de técnicas GBL, es el desarrollo de habilidades y competencias transversales específicas (directamente o no relacionadas con su campo profesional) (Menon y Romero 2020; J. Sanchez-Martín, Zamora-Polo y col. 2017; Zamora-Polo, Luque-Sendra y col. 2019). Dentro de las actividades GBL, las escape rooms en el ámbito educativo están teniendo un éxito significativo. Una sala de escape o escape room (ER) se puede definir como una actividad recreativa en la que un grupo de personas es encerrado en una o más habitaciones de las que deben escapar en un tiempo limitado resolviendo una serie de acertijos, cuestionarios y puzles (Zhang y col. 2017). Aunque las salas de escape se crearon con fines recreativos (http://realdgame.jp/event/nazotokinoutage.html 2017) cuando se utilizan con fines educativos son capaces de ayudar en el desarrollo de habilidades y competencias transversales como el trabajo en equipo, el pensamiento lateral y crítico, la comunicación, el trabajo bajo presión, 
etc. (Pan, Lo y Neustaedter 2017; Shakeri y col. 2017). En la literatura se pueden encontrar algunas aplicaciones de las ER en el contexto educativo (Fotaris y Mastoras 2019; Bassford y col. 2016; Batzogiannis, Hatzikraniotis y Papadopoulos 2018), aunque la mayoría de ellas han sido desarrolladas en la educación superior.

Las salas de escape se basan en la implementación de una temática y una narrativa que sirve como hilo conductor de la actividad. Las temáticas pueden ser muy variadas y se pueden adaptar a muchas áreas de la educación superior. La elección del tema conlleva la adaptación y diseño de los puzles y las pruebas que se realizarán durante la actividad. Hay una amplia variedad de rompecabezas que se pueden incorporar en una sala de escape (Shakeri y col. 2017). También es importante tener en cuenta el espacio donde se desarrollará la actividad y cómo se implementarán los puzles (Clarke y col. 2018). Si bien es evidente que la experiencia ïn situ"potencia la positividad de la actividad y refuerza las habilidades que se pretende desarrollar, esto no siempre es posible por no disponer de los recursos necesarios (espacio físico suficiente, trabajar en pequeños grupos y materiales empleados). La falta de estos recursos ha motivado, incluso antes del estallido de la pandemia, la aparición de escape rooms virtuales a través de aplicaciones informáticas (Mystakidis, Cachafeiro y Hatzilygeroudis 2019), que se pueden adaptar fácilmente en las plataformas educativas con las que cuentan las universidades en la actualidad.

El éxito de actividades como las escape rooms se basa en la gran cantidad de emociones positivas que experimentan los estudiantes cuando participan (alegría, interés, etc.) y que favorecen el proceso de enseñanza-aprendizaje (Jeong, Canada-Canada y Gonzalez-Gomez 2018). Se ha comprobado que a mayor rendimiento emocional positivo, mejores calificaciones académicas se obtienen (Mellado y col. 2014), por lo que este tipo de actividades son una excelente manera de mejorar la motivación del alumno STEM Martínez-Borreguero y col. 2018. Se ha probado que la adquisición de conceptos científicos difíciles mejora mucho cuando se introducen a través de experiencias recreativas que potencien la motivación y el desempeño emocional de los estudiantes (Sanchez-Martin, CanadaCanada y Davila-Acedo 2018; Buckley y Doyle 2016; Mullins y Sabherwal 2018).

El estallido de la pandemia ha brindado la oportunidad para que estas nuevas metodologías educativas y GBL demuestren que pueden ser de gran utilidad para mejorar el desempeño y la motivación de los estudiantes. En este artículo presentamos las actividades que se han llevado a cabo para implementar una escape room en la asignatura de Órbitas, satélites y relatividad, con el fin de reforzar los conocimientos adquiridos y los resultados que se han obtenido.

\section{Objetivos}

Se propone como objetivo:

1. la creación de una escape room mediante los elementos digitales disponibles en las plataformas educativas de la UPV, principalemnte PoliformaT (SAKAI).

2. incrementar la motivación y participación de los estudiantes, mediante el juego con los elementos principales que aparecen en el temario de la asignatura.

3. potenciar las habilidades transversales de resolución de problemas y trabajo colaborativo. 


\section{Desarrollo de la innovación}

En esta sección se describirá la aplicación de una escape room en la asignatura optativa de Órbitas, Satélites y Relatividad, perteneciente al Grado de Ingeniería Aeroespacial de la Escuela Técnica Superior de Ingeniería del Diseño (ETSID) en la Universitat Politècnica de València (UPV), con el fin de aumentar la motivación de los estudiantes, que seguían la asignatura online empleando PoliformaT (SAKAI) y TEAMS (Microsoft).

PoliformaT es una plataforma educacional que se emplea en la UPV para la comunicación e interacción de los estudiantes y profesores. Esta plataforma permite la realización de exámenes online, chat, envío de correos electrónicos, compartir recursos educativos, etc. La herramienta Exámenes permite realizar diferentes tipos de preguntas, como por ejemplo, las preguntas de elección múltiple, de respuesta numérica, de subida de archivos, respuesta abierta, etc. Además, permite configurar los tests o exámenes de forma que un número limitado de estudiantes lo pueda realizar, las fechas de disponibilidad, el tiempo de realización de la prueba, el número de intentos permitido o el acceso a través de una contraseña. En la Figura 1 se pueden ver alguna de estas características.

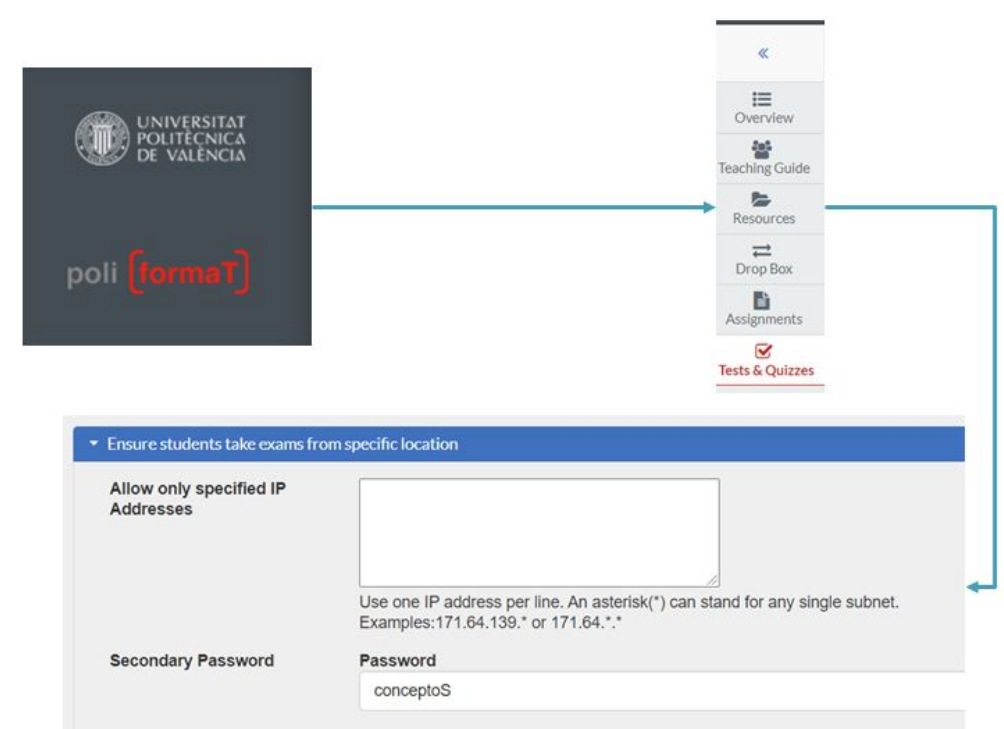

Fig. 1: PoliformaT una de las plataformas educativas empleada por la Universitat Politècnica de València. Permite la creación de exámenes con contraseña y limitación de tiempo.

Por otra parte, la plataforma TEAMS actuó como comunicador entre los integrantes del grupo. Entre las fortalezas de esta plataforma se encuentra la de posibilitar la creación de grupos de interacción y la comunicación entre sus integrantes de una forma fluida.

Estas herramientas han permitido configurar un práctica online con los elementos típicos de una ER, tales como una temática, una narrativa y un tiempo limitado, pero con la diferencia que en esta práctica se ha primado la colaboración en grupos.

La narrativa del escape room online se basó en el temario de la propia asignatura: el objetivo era rescatar a un compañero que se había perdido en una nave espacial cuando viajaba a la 
Luna, y para ello se debía diseñar una trayectoria adecuada en un corto período de tiempo. Las características del vehículo de rescate limitaban las acciones a realizar, por lo que los estudiantes debían diseñar una estrategia que optimizara el tiempo y los DeltaV utilizados (aunque, en una primera aproximación, el tiempo no es un factor determinante) .

Al final de la actividad, que se considera terminada en el momento en que el grupo encuentra una solución factible al problema presentado, los estudiantes comparan sus soluciones con las soluciones obtenidas por el resto de grupos, con el fin de obtener de una forma razonada la mejor de todas las estrategias, o bien modificar las soluciones para intentar mejorar las soluciones obtenidas. Esta última actividad, tiene la ventaja de que permite a los grupos interactuar con mayor confianza, es decir, el hecho de tener una solución permite defenderla y compararla frente a sus compañeros. Esto permite una interacción mucho más focalizada y permite profundizar en los conceptos que los estudiantes deben dominar al finalizar el curso. Cabe mencionar que el uso de la plataforma PoliformaT no limita en absoluto la aplicabilidad de esta metodología, ya que la mayoría de plataformas educativas permiten la implementación de todos los elementos mencionados.

La actividad comienza dividiéndose en grupos de entre cuatro a seis estudiantes, dependiendo del número total de estudiantes. La contraseña que permitió el inicio de la actividad es proporcionada por los profesores al inicio de la actividad. Nada más iniciada la actividad se presenta a los alumnos la situación en la que se encuentra perdido en el espacio un compañero (que pertenece a la clase, para incrementar la implicación emocional en la actividad, y que participa en el proceso de obtención de la solución) junto con los datos técnicos necesarios.

Las pruebas se basan en preguntas y acertijos que permiten combinar elementos lúdicos que no pertenecen al ámbito universitario (como buscar información en internet, encontrar secuencias de números) con elementos más propios de la asignatura (como problemas relacionados con la asignatura) como el número de impulsos necesarios para el rendezvous con la nave del compañero, el DeltaV consumido y la optimización de la trayectoria.

La sala de escape se dividió en cuatro actividades:

1. establecer la posición de la nave espacial perdida

2. establecer una nave espacial de rescate adecuada y su posición y características

3. desarrollar la estrategia adecuada para el rendezvous

4. evaluar y comparar los resultados de los grupos para obtener una solución óptima

En la última fase, cada grupo presenta el número de impulsos, el tiempo de vuelo y el total DeltaV para evaluar y comparar sus propios resultados. El objetivo ahora es potenciar el trabajo colaborativo estudiando lo mejor de las trayectorias y/o combinarlas para encontrar una estrategia que mejore las presentadas.

El tiempo límite establecido fue de una hora, más 15 minutos adicionales para compartir posibles soluciones entre los estudiantes. En la Figura 2 se puede ver el plan de la actividad, detallando alguno de los pasos realizados. 


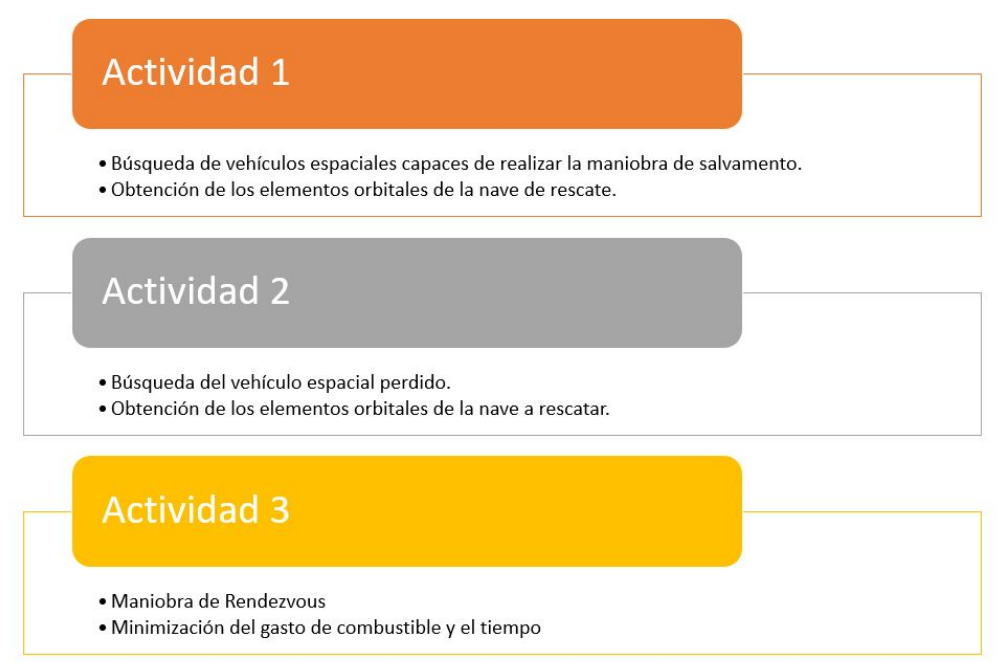

Fig. 2: Plan de actividad. La escape room consistió en tres actividades principalmente. La primera actividad es la búsqueda del vehículo espacial necesario para hacer el rescate. La segunda actividad consiste en localizar el vehículo perdido y la tercera consiste en el diseño de una estrategia de rescate para rescatar la nave perdida.

\section{Resultados}

Los resultados se dividen en dos categorías: la primera reflejará los resultados obtenidos por los estudiantes desde un punto de vista académico, la segunda se centrará en la percepción de los estudiantes de las habilidades transversales y las opiniones sobre la actividad.

\subsection{Resultados académicos}

La solución al problema presentado a los estudiantes es la combinación de maniobras impulsivas consecutivas en el apogeo o la combinación de maniobras impulsivas en el apogeo y perigeo (bielípticas). Los estudiantes, a la hora de la realización de la práctica, conocían estas maniobras por separado, pero no la concatenación de ellas. El ejercicio consistió en asegurar que los estudiantes fueran capaces de encontrar una sucesión de maniobras capaces de llegar a una órbita concreta con una limitación significativa de combustible y maniobrabilidad (ya que de lo contrario, sin estas limitaciones, la maniobra podría ser directa). También con la condición de que el DeltaV se minimice, los estudiantes deben realizar las maniobras impulsivas solo en el apogeo o perigeo.

En la Figura 3a se puede ver la maniobra presentada a los estudiantes como una maniobra de Hohmann, mientras que en la Figura 3 b y $3 \mathrm{c}$ se presentan posibles soluciones al problema planteado. Al finalizar la actividad, todos los grupos pudieron alcanzar la órbita solicitada, utilizando las técnicas presentadas en la Figura 3. En algunos casos, los estudiantes fueron capaces de combinar estas técnicas. Ha de tenerse en cuenta que en la ER sólo tenían que alcanzar la órbita para rescatar al compañero, sin limitación en el número de impulsos, más allá de las limitaciones que imponían la cantidad de combustible disponible y de la cantidad de energía generada en cada impulso. Los estudiantes, sin embargo, entendieron que esto tenía que hacerse con el menor número de impulsos, por lo que varios grupos trabajaron en un algoritmo implementado en MATLAB para estudiar la 


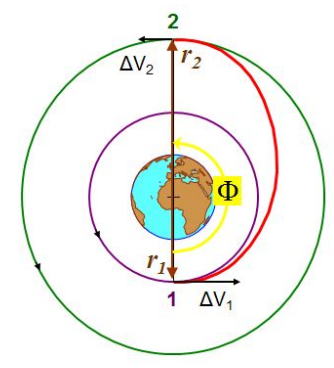

a) Hohmann

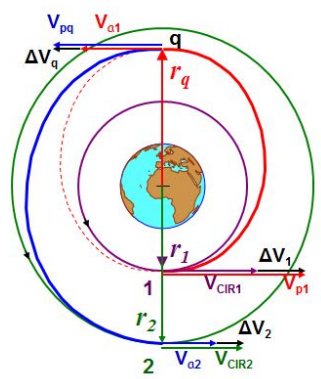

b) Bi-elliptic

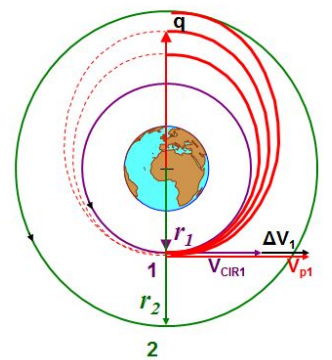

c) Apogee maneuver

Fig. 3: (a) Maniobra de Hohmann. (b) Maniobra Bi-elíptica consistente en tres impulsos para llegar a una órbita circular externa. (c) Maniobras de cambio de altura del apogeo.

solución que redujera estos impulsos. La solución óptima que minimiza el tiempo de vuelo y el número de impulsos consistía en una maniobra con seis impulsos que combinaba transferencia bi-elíptica y cambio de apogeo.

Tabla 1: Resultados de la actividad

\begin{tabular}{llll}
\hline Grupo & Impulsos & Tipo & $\Delta V(\mathrm{~km} / \mathrm{s})$ \\
\hline Grupo 1 & 6 & Bi-elíptica & 6.06 \\
Grupo 2 & 6 & Bi-elíp. + cambio apogeo & 5.92 \\
Grupo 3 & 7 & Cambio apogeo & 6.16 \\
Grupo 4 & 7 & Cambio apogeo & 6.16 \\
Grupo 5 & 6 & Bi-elíp. + cambio apogeo & 5.92 \\
Grupo 6 & 6 & Bi-elíp. + cambio apogeo & 5.92 \\
\hline
\end{tabular}

Como se puede ver en los resultados, tres grupos lograron el menor número de impulsos combinando las técnicas vistas anteriormente. La última etapa de la actividad consistió en que los diferentes grupos compartieran sus resultados y compararan los valores obtenidos. Entre todos tenían que dar el mejor resultado. Para lograrlo, tuvieron que discutir las maniobras seleccionadas y verificar que los cálculos fueran correctos. Esta fase intentó promover la colaboración activa de los estudiantes y su participación en el proceso de aprendizaje. 


\subsection{Opinión de los estudiantes}

Un punto importante en la evaluación de la actividad es la opinión de los estudiantes sobre los diferentes elementos empleados en la actividad. En primer lugar, nos centraremos en la apreciación de la motivación y el interés de los alumnos por la asignatura. El uso de elementos de gamificación tuvo como objetivo aumentar la motivación para el aprendizaje de la asignatura Órbitas, Satélites y Relatividad. Se aplicó una encuesta a los estudiantes en la que puntuaron de uno a cinco (siendo uno la puntuación más baja o más negativa y cinco la puntuación más alta o más positiva) los siguientes ítems:

- motivación por la asignatura antes de la actividad

- motivación por la asignatura después de la actividad

- percepción sobre el interés de la asignatura

- desarrollo de la habilidad de trabajo colaborativo

- desarrollo de la habilidad de resolución de problemas

- diversión durante la actividad

- dificultad de la actividad

En relación a la evaluación de los ítems "trabajo colaborativoz resolución de problemas", los estudiantes evaluaron su percepción sobre su mejora en estas habilidades. Las respuestas deben considerarse con cuidado, ya que la respuesta positiva mayoritaria que se obtuvo está influenciada por el éxito de los grupos en lograr el objetivo de la sala de escape, y es además tan sólo una percepción desde el punto de vista personal. Una investigación sobre la evolución de estas habilidades se está llevando a cabo actualmente. Sin embargo, incluimos dichos resultados como un indicador subjetivo de la valoración que los estudiantes tienen de estas competencias.

En la siguiente tabla 2 se presenta un resumen numérico de los resultados obtenidos en cada ítem.

Si hacemos una comparación de las medias obtenidas entre los ítems motivación después y motivación antes de la actividad, mediante una prueba de hipótesis, donde la hipótesis nula indica que las medias son iguales y la hipótesis alternativa que la media de la motivación después es mayor que la motivación antes, con un nivel de significación de alpha $=0,05$, se obtiene un p-valor obtenido de 0.99 , por lo que aceptamos la hipótesis alternativa, es decir, ha habido un aumento en la motivación de los estudiantes.

En cuanto a las opiniones de los alumnos sobre si han disfrutado de la actividad, se observa en la Figura 4, que el $100 \%$ ha valorado la actividad con un 4 o un 5. La opinión sobre la dificultad es más baja. Para algunos estudiantes la dificultad fue elevada. Cuando se le preguntó qué elementos dificultaban la actividad, el $97 \%$ respondió que la limitación de tiempo había ejercido una gran presión sobre la actividad.

Cuando se les preguntó a los estudiantes si tenían la sensación de haber aprendido de la actividad, las respuestas fueron muy positivas (ver Figura 5). La sensación general fue de haber obtenido un aprendizaje profundo sobre la optimización de las trayectorias de las órbitas. 
Tabla 2: Resumen de los resultados. Todos los items fueron evaluados de 1 a 5 , donde 1 significaba la más baja o la más negativa de las opiniones y 5 la más alta o positiva. La tabla muestra los estadísticos principales (Media, desviación típica (sd), cuartiles).

\begin{tabular}{|c|c|c|c|c|c|c|c|}
\hline Item & Media & $\mathrm{Sd}$ & Q $0 \%$ & Q $25 \%$ & Q $50 \%$ & Q $75 \%$ & Q $100 \%$ \\
\hline Colaboración & 4.54 & 0.66 & 3 & 4 & 5 & 5 & 5 \\
\hline Dificultad & 3.96 & 0.81 & 3 & 3 & 4 & 5 & 5 \\
\hline Diversión & 4.63 & 0.49 & 4 & 4 & 5 & 5 & 5 \\
\hline Aprendizaje & 4.71 & 0.46 & 4 & 4 & 5 & 5 & 5 \\
\hline $\begin{array}{l}\text { Motivación } \\
\text { después }\end{array}$ & 4.75 & 0.44 & 4 & 4 & 5 & 5 & 5 \\
\hline $\begin{array}{l}\text { Motivación } \\
\text { antes }\end{array}$ & 4.08 & 0.83 & 2 & 4 & 4 & 5 & 5 \\
\hline $\begin{array}{l}\text { Resolución } \\
\text { problemas }\end{array}$ & 4.54 & 0.51 & 4 & 4 & 5 & 5 & 5 \\
\hline
\end{tabular}

La sensación de haber mejorado en las competencias transversales relacionadas con el trabajo en equipo y la resolución de problemas es generalizada (ver Figura 6). Esto, como hemos comentado antes, puede deberse a la sensación de haber podido resolver la actividad propuesta en clase. Sería necesario estudiar si esta sensación se traduce en mejores resultados en actividades posteriores o se mantiene en el tiempo.

\section{Conclusiones}

El objetivo de este proyecto fue la implementación de una actividad de escape room virtual que simulara una posible situación de emergencia en el espacio, utilizando vehículos espaciales, y que pudiera resolverse mediante el uso de mecánica orbital. En concreto, la ER fue diseñada para reforzar las habilidades transversales de pensamiento crítico, trabajo en equipo y resolución de problemas.

Los estudiantes percibieron la actividad con una actitud sumamente positiva, y sus percepciones del impacto de esta actividad en las competencias de trabajo en grupo y la resolución de problemas es, en general, de mejora. Esta actividad también ha permitido un refuerzo de los conocimientos necesarios en la propia asignatura. Es decir, el empleo de esta tipo de actividad, además de mejorar la motivación en la asignatura (Mullins y Sabherwal 2018), tiene la capacidad potencial de mejorar el rendimiento académico de los estudiantes (Mellado y col. 2014; Sanchez-Martin, Canada-Canada y Davila-Acedo 2018). De los resultados obtenidos en la encuesta posterior a la actividad y del feedback proporcionado por los estudiantes, se puede deducir que esta actividad alcanzó las expectativas deseadas. Sin embargo, algunos estudiantes manifestaron que habían sufrido cierto estrés debido a las limitaciones de tiempo y las pruebas realizadas. En futuras revisiones, esta limitación de tiempo debe tenerse en cuenta en función de la dificultad de la prueba, y equilibrarse si es necesa- 


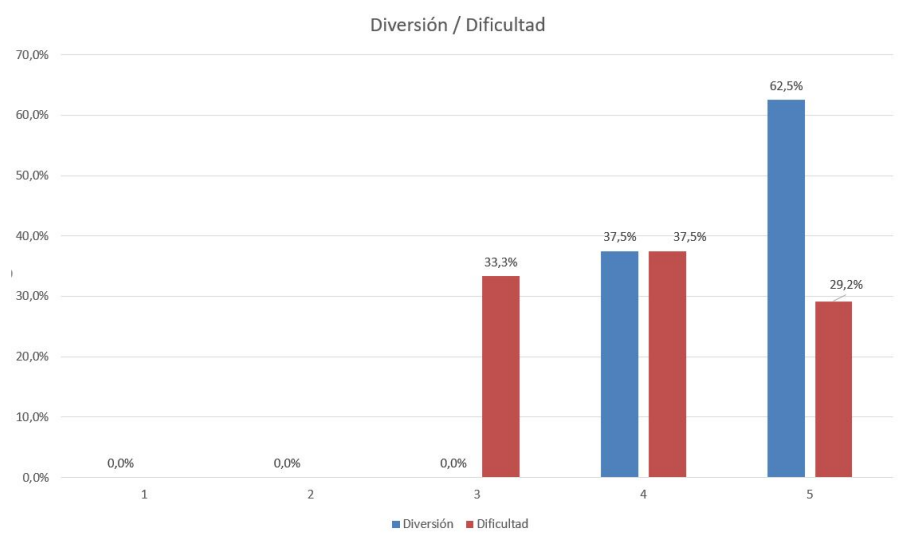

Fig. 4: Resultados de las opiniones de los estudiantes respecto a la diversión y dificultad.

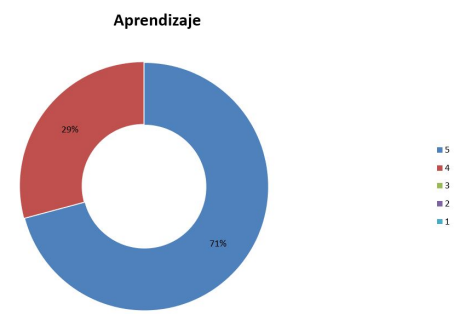

Fig. 5: Resultados de las opiniones de los estudiantes respecto al aprendizaje efectivo de la actividad.

rio, de modo que se minimicen los niveles de estrés en favor de la integración y la participación. Los autores, sin embargo, piensan que un nivel de estrés equilibrado mejora la concentración durante la actividad, por lo que no están a favor de aumentar excesivamente la limitación de tiempo.

Los resultados aquí presentados son muy positivos y corroboran que el uso de elementos de gamificación en el aula tiene la capacidad de mejorar las habilidades de los estudiantes, al tiempo que mejora la motivación y el interés por el conocimiento en general (lo que viene corroborado en la literatura: Bassford y col. 2016; Martínez-Borreguero y col. 2018; Sanchez-Martin, Canada-Canada y Davila-Acedo 2018; Buckley y Doyle 2016).

La preparación de este tipo de actividades puede llevar bastante tiempo, ya que la prueba y los cuestionarios deben cubrir diferentes conceptos necesarios para los estudiantes y, al mismo tiempo, deben presentar una narrativa atractiva y envolvente que sumerja al estudiante en la actividad. Sin embargo, el diseño de pruebas, acertijos es más sencillo, ya que son muchos los problemas y acertijos que los profesores utilizan en clase, muchas veces desprovistos de la narrativa adecuada, que pueden implementarse en una sala de escape o en una actividad de gamificación.

Los elementos tecnológicos utilizados en esta actividad se pueden encontrar en prácticamente cualquier plataforma educativa y se pueden implementar fácilmente en el diseño de actividades como escape rooms. En la actualidad, y si bien la enseñanza a distancia es un elemento importante en el 


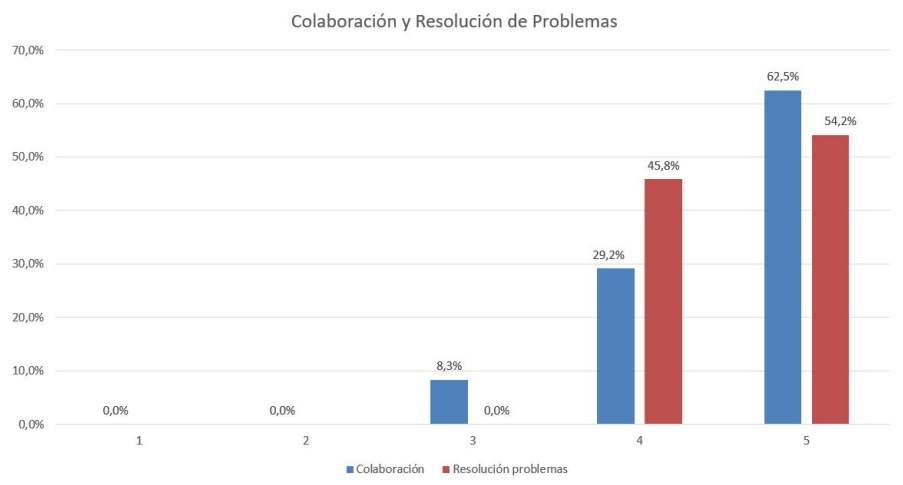

Fig. 6: Resultados de las opiniones de los estudiantes respecto a las competencias de colaboración en grupo y resolución de problemas.

diseño de asignaturas, los recursos tecnológicos juegan un papel fundamental en la transmisión del conocimiento. Es posible que aun cuando la situación se normalice luego de esta pandemia, ciertos recursos y metodologías no dejarán de ser utilizados, ya que implican un avance significativo en la descentralización del proceso de enseñanza-aprendizaje con respecto al docente y un equilibrio a favor de la estudiante.

Actividades como la presentada tienen una fácil implementación que no puede competir con las necesidades físicas de realizar la actividad in situ en el aula (Mystakidis). La tendencia al ahorro del tiempo y los materiales asegura un futuro para los recursos en línea de la enseñanza a distancia. Y la creación de contenido online tendrá un impacto en la ciencia de los próximos años, ya que este material estará a disposición de los estudiantes para marcar su ritmo de aprendizaje y ayudarles a profundizar en sus conocimientos.

\section{Referencias bibliográficas}

Bassford, M.L. y col. (2016). "CrashEd - a live immersive, learning experience embedding STEM subjects in a realistic, interactive crime scene". En: Res. Learn. Technol. 24(1), págs. 30089-30093.

Batzogiannis, I., E. Hatzikraniotis y A. Papadopoulos (2018). "Enhancing students' motivation towards STEM by co-creating an escape room". En: INTED 2018 Proceedings, págs. 3293-3300.

Borrego, C. y col. (2017). "Room escape at class: escape games activities to facilitate the motivation and learning in computer science". En: J. Technol. Sci. Educ. 7(2), págs. 162-171.

Buckley, P. y E. Doyle (2016). "Gamification and student motivation". En: Interact. Learn. Environ. 24(6), págs. 1162-1175.

Bybee, R.W. (2013). The Case for STEM Education: Challenges and Opportunities. NSTA press, Arlington, Virginia, USA. 
Clarke, S. y col. (2018). "Escaped: adapting live action, interactive games to support higher education teaching and learning practices". En: Lecture Notes in Computer Science (Including Subseries Lecture Notes in Artificial Intelligence and Lecture Notes in Bioinformatics).

Deterding, S. (2012). "Gamification: design for motivation". En: Interactions 19(4), págs. 14-17.

Deterding, S. y col. (2011). "From game design elements to gamefulness: defining "gamification ". En: Proceedings of the 15th International Academic MindTrek Conference: Envisioning Future Media Environments, págs. 9-15.

Ebner, M. y A. Holzinger (2007). "Successful implementation of user-centered game based learning in higher education: an example from civil engineering". En: Comput. Educ. 49(3), págs. 873-890.

Fotaris, P. y T. Mastoras (2019). "Escape rooms for learning: a systematic review". En: Proceedings of the 13th European Conference on Games Based Learning ECGBL, págs. 9-18.

Hamari, J. y col. (2016). "Challenging games help students learn: an empirical study on engagement, flow and immersion in game-based learning". En: Comput. Hum. Behav. 54, págs. 170-179.

http://realdgame.jp/event/nazotokinoutage.html, Available online: (2017). SCRAP, Real Escape Game project first series.

Jeong, J., F. Canada-Canada y D. Gonzalez-Gomez (2018). "The study of flipped-classroom for pre-service science teachers". En: Educ. Sci. 8(4), págs. 163-171.

De-Marcos, L. y col. (2014). "An empirical study comparing gamification and social networking on e-learning". En: Comput. Educ. 75, págs. 82-91.

Martínez-Borreguero, G. y col. (2018). "Recreational experiences for teaching basic scientific concepts in primary education: the case of density and pressure". En: Eurasia J. Math. Sci. Technol. Educ. 14(12), em1616.

Mellado, V. y col. (2014). "Las emociones en la enseñanza de las ciencias / Emotions in science teaching". En: Enseñanza de las Ciencias 32(3), págs. 11-36.

Menon, D. y M. Romero (2020). "Game mechanics supporting a learning and playful experience in educational escape games. In Global Perspectives on Gameful and Playful Teaching and Learning". En: IGI Global, págs. 143-162.

Mullins, J.K. y R. Sabherwal (2018). "Beyond enjoyment: a cognitive-emotional perspective of gamification". En: Proceedings of the 51st Hawaii International Conference on System Sciences, págs. 1237-1246.

Mystakidis, S., E. Cachafeiro e I. Hatzilygeroudis (2019). "Enter the Serious E-Scape Room: A Cost-Effective Serious Game Model for Deep and Meaningful E-Learning". En: 2019 10th International Conference on Information, Intelligence, Systems and Applications (IISA), págs. 1-6. 
Pan, R., H. Lo y C. Neustaedter (2017). "Collaboration, awareness, and communication in real-life escape rooms". En: DIS 2017 - Proceedings of the 2017 ACM Conference on Designing Interactive Systems, págs. 1353-1364.

Papastergiou, M. (2019). "Digital Game-Based Learning in high school Computer Science education: impact on educational effectiveness and student motivation". En: Comput. Educ. 52(1), págs. 1-12.

Rodrigues, L.F., A. Oliveira y H. Rodrigues (2019). "Main gamification concepts: a systematic mapping study". En: Heliyon 5(7), e01993.

Ross, R. y C. Bell (2019). "Turning the classroom into an escape room with decoder hardware to increase student engagement". En: 2019 IEEE Conference on Games (CoG), págs. 1-4.

Sanchez-Martin, J., F. Canada-Canada y M.A. Davila-Acedo (2018). "Emotional responses to innovative Science teaching methods: acquiring emotional data in a General Science teacher education class". En: J. Technol. Sci. Educ. 8(4), págs. 346-359.

Sanchez-Martín J.and Canada-Canada, F. y M.A. Davila-Acedo (2017). "Just a game? Gamifying a general science class at university: collaborative and competitive work implications". En: Think. Skills Creativ. 26, págs. 51-59.

Sanchez-Martín, J., M. Corrales-Serrano y col. (2020). "Exit for success. Gamifying science and technology for university students using escape-room. A preliminary approach". En: Heliyon 6, $\mathrm{e} 04340$.

Sanchez-Martín, J., F. Zamora-Polo y col. (2017). "Innovative education tools for developing ethical skills in university science lessons. The case of the moral cross dilemma. Ramon Llull". En: J. Appl. Ethics 8, págs. 225-245.

Shakeri, H. y col. (2017). "Escaping together: the design and evaluation of a distributed real-life escape room." En: Proceedings of the Annual Symposium on Computer-Human Interaction in Play. ACM., págs. 115-128.

Warmelink, H. y col. (2020). "Gamification of production and logistics operations: status quo and future directions". En: J. Bus. Res. 106, págs. 331-340.

Zamora-Polo, F., M. Corrales-Serrano y col. (2019). "Nonscientific university students training in general science using an active-learning merged pedagogy: gamification in a flipped classroom". En: Educ. Sci. 9(4), págs. 297-315.

Zamora-Polo, F., A. Luque-Sendra y col. (2019). "Conceptual framework for the use of building information modeling in engineering education". En: Int. J. Eng. Educ. 35(3), págs. 744-755.

Zhang, F. y col. (2017). "Employing a user-centered design process to create a multiplayer online escape game for older adults". En: Proceedings of the Annual Symposium on Computer-Human Interaction in Play, Zhou J, S.G. (Ed.), págs. 296-307. 